\title{
Modelo del proceso jerárquico análitico para optimizar la localización de una planta industrial
}

\begin{abstract}
RESUMEN
La localización de una Planta Industrial, es el proceso de elegir un lugar geográfico para realizar las operaciones de una empresa. Para determinar la mejor localidad, es necesario realizar una evaluación de los factores que condicionan la selección. Se consideran dos grupos de factores: Cuantitativos, que son fáciles de cuantificar y Cualitativos, que son menos tangibles, difíciles de cuantificar, la evaluación de estos últimos depende de la apreciación del analista, manteniendo un tinte de subjetividad, lo que hace que ningún modelo utilizado garantice que el lugar elegido sea el óptimo.

Para optimizar la calificación de los factores, se propone utilizar el modelo de PROCESO JERÁRQUICO ANALÍTICO (PJA), con una escala de calificación adecuada, que asegura la consistencia de la misma y permite la utilización de un software (Expert Choice).

El presente estudio expone y demuestra el aporte del Proceso Jerárquico Analítico (PJA) en la tarea de optimización de la localización de una planta industrial, en comparación con otros métodos.
\end{abstract}

Palabras clave: Localización de planta, Proceso Jerárquico Analítico, Factores Locacionales

Analytical hierarchy process MODEl to OPTIMIZE THE LOCATION OF AN INDUSTRIAL PLANT

\section{ABSTRACT}

The location of an industrial plant, is the process of choosing a geographical location to perform the operations of a company. To determine the best location, you must perform an assessment of the factors affecting the selection. Two groups of factors are considered: Quantitative, which are easy to quantify and Qualitative that are less tangible, hard to quantify, evaluation of the latter depends on the judgment of the analyst, keeping a tinge of subjectivity, making no model used to ensure that the venue is optimal.

To optimize the classification of factors, it is proposed to use the model of Analytic Hierarchy Process (AHP) with a proper rating scale, which ensures consistency of it and allows the use of a software (Expert Choice).

This study presents and demonstrates the contribution of the Analytic Hierarchy Process (AHP) in the task of optimizing the location of an industrial plant, compared with other methods.

Keywords: Plant location, Analytic Hierarchy Process, locational factors

\section{INTRODUCCIÓN}

El concepto de localización de una planta industrial, se refiere a la ubicación de una nueva unidad productora, de tal manera que se logre la máxima rentabilidad del proyecto o el mínimo de los costos unitarios. La localización de las instalaciones de una empresa tiene impacto significativo en los costos de operación de la compañía, los precios que esta cobra por los productos y servicios y la capacidad que tiene para competir en el mercado y penetrar nuevos segmentos de clientes.

Se consideran dos tipos de factores: a) Cuantitativos, que son fáciles de cuantificar, como por ejemplo el costo de mano de obra, costo de materiales, costo de transporte, demanda, etc. y b) Cualitativos, difíciles de cuantificar, por ejemplo impacto ambiental, actitud de la comunidad, clima, vivienda, centros de capacitación, seguridad, etc.

Existen modelos que permiten evaluar los factores locacionales, facilitando la evaluación de los factores cuantitativos por sus cantidades. La evaluación de los factores cualitativos lo hacen por su preferencia o asignándoles un puntaje de acuerdo a la apreciación del analista, lo que hace que ningún modelo utilizado garantice que el lugar elegido sea el óptimo.

\section{SITUACIÓN PROBLEMÁTICA}

\subsection{ANTECEDENTES}

Resendiz (2009) desarrolla una tesis para optar el título de Ingeniero Industrial, en la cual se propone una solución para la reducción de la basura orgánica generada en una universidad mexicana mediante la implementación de una planta productora de lombricomposta, para ello recurre a la propuesta de Thomas L. Saaty llamada Proceso Jerárquico Analítico,

García, María (2009), aplica el Proceso Jerárquico Analítico en su tesis doctoral "Métodos para la comparación de alternativas mediante un Sistema de Ayuda a la Decisión (S.A.D.) y Soft Computing"

\footnotetext{
* Mg. Ingeniería Industrial. Docente principal del Dpto. de Producción y Gestión Industrial de la Facultad de Ingeniería Industrial. E-mail: jasalasb@hotmail.com

** Ing. Químico. Docente Asociado del Dpto. de Análisis y de Diseño de Procesos de la Facultad de Química e Ing. Química. UNMSM. E-mail: maxleyvac2@hotmail.com

*** Mg. Desarrollo y Defensa Nacional. Mg. Estrategia Marítima. Docente Escuela de Marina Mercante. Escuela Superior de Guerra Naval. CITEN-UAP. E-mail: aecf@mixmail.com
} 
Cock German y López Juan (2009), en "Lúdica aplicada al problema de localización de planta física utilizando Analytic Hierarchy Process (AHP), y Solver Excel".

Pérez Víctor, Camargo Isis, Caballero Rafael y González Mercedes (2008), aplican el Proceso Jerárquico Analítico de Saaty, en "Selección Multicriterio de Nuevos Productos Turísticos en Pinar del Río Cuba".

Toskano, Bruno (2005) aplica el método de Proceso Jerárquico Analítico para optimizar la selección de proveedores, en sus tesis para Licenciatura en Investigación de Operaciones, en la UNMSM.

Avila, Ruth (2000), en "El PJA (Proceso Jerárquico Analítico) y su aplicación para determinar los usos de las tierras". El trabajo pretende brindar a los lectores, orientaciones generales sobre el método PJA, a fin de priorizar escenarios de los usos de las tierras, dentro del proceso de toma de decisión en el Municipio de Concordia en Brasil.

\subsection{PLANTEAMIENTO DEL PROBLEMA}

La localización de la instalación es el proceso de elegir un lugar geográfico para realizar las operaciones de una empresa. En la selección, se deben evaluar los factores locacionales o condicionantes, en las localidades factibles.

Los factores de localización los consideramos en dos grupos:

a) Factores Cuantitativos: son aquellos fáciles de cuantificar, se expresan por sus respectivas cantidades. Ej.: mano de obra, materia prima, transporte, demanda, terreno, capital, costo de servicios, impuestos y seguros, etc.

b) Factores Cualitativos, que son menos tangibles, Ej.: impacto ambiental, educación, vivienda, clima, actitud de la comunidad, políticas de gobierno, protección contra incendios, seguridad, ambiente laboral, calidad de vida, competencia, etc.

\section{DEFINICIÓN DEL PROBLEMA}

¿Es posible establecer un modelo de calificación para la evaluación de los factores cualitativos, garantizando la optimización del proceso de localización de una planta industrial?

\subsection{JUSTIFICACION E IMPORTANCIA}

Se ha determinado que una de las decisiones más importantes para el funcionamiento de una industria, es el de la localización de planta, ya que de la buena ubicación de ella dependerá el éxito o el fracaso de una empresa.

Las decisiones de localización tienen naturaleza de largo plazo y es por ello sumamente importante una adecuada evaluación de las localidades, para asegurar que la comunidad elegida sea la óptima.

\subsection{PLANTEAMIENTO DE LAS HIPÓTESIS}

\section{Hipótesis General}

El modelo del Proceso Jerárquico Analítico, en la calificación para la evaluación de los factores cualitativos, optimiza el proceso de localización de una planta industrial

\subsection{DEFINICIÓN DE VARIABLES}

\section{VARIABLE INDEPENDIENTE (X)}

Modelo del Proceso Jerárquico Analítico: Su aplicación disminuye el nivel de subjetividad en la evaluación o calificación de los factores cualitativos en una localización de una planta industrial, optimizándola.

Indicadores:

- Calificación de factores.

- Priorización por factores y localidades

- Nivel de subjetividad

\section{VARIABLE DEPENDIENTE ( $Y$ )}

Proceso de localización de una planta industrial: Se optimiza el proceso de selección al aplicar el modelo de Proceso Jerárquico Analítico, eligiendo la localidad que garantice la rentabilidad de la empresa.

Presenta los siguientes indicadores:

- Ubicación de planta industria

\section{MARCO TEÓRICO}

Desde comienzos del siglo XIX se intenta buscar un modelo teórico que explique la localización industrial. Weber (1909) fue uno de los pioneros en el desarrollo de una teoría pura sobre la localización industrial en el espacio. Este científico regionalista hace referencia a los recursos 0 materias primas que sólo existen o se concentran en lugares determinados. Aceptó que la localización de la planta productiva está influenciada por otros factores (mano de obra) que hacen más competitiva a la industria, pero supuso que dentro de una región los costos de ese factor eran constantes. 
Entonces la característica principal de su modelo se encuentra en un patrón espacial de costos fijos y mano de obra ubicua (que está presente a un mismo tiempo en todas partes)

Según Weber, citado por Vasquez (1988) la ubicación de una planta industrial está relacionada con cuatro factores fundamentales: la distancia a los recursos naturales, la distancia al mercado, los costes de la mano de obra y las economías de aglomeración.

Greenhut (1957), citado por Hernández (2009), hace dos aportaciones interesantes, en las que introduce el papel de la toma de decisiones y las variables económicas, con el fin de ajustarse aún mas a la realidad. Dijo que cada competidor adopta una estrategia entre las varias posibles, para poder elegir la localización.

\subsection{LOCALIZACIÓN DE PLANTA}

Se entiende por Localización de Planta, el lugar elegido por el empresario para situarla y desarrollar su capacidad productiva. El problema de la localización de planta, puede definirse como la determinación de aquel lugar que, considerados todos los factores, ocasionará el costo mínimo del producto a fabricar, con el objeto de mantener los máximos beneficios empresariales, proporcionando a su vez la máxima satisfacción posible a los clientes.

Una vez decidida la ubicación, el empresario no podrá rectificar sin asumir un coste importante, que supondrá pérdidas importantes (e incluso no soportables) por parte de la empresa.

\subsection{MÉTODOS O MODELOS DE LOCALIZACIÓN DE PLANTA}

En el estudio se han considerado los siguientes métodos o modelos de localización de planta:

a) Método del ranking de factores

b) Método de calificación del factor cualitativo

c) Método del análisis dimensional

d) Método de Brown y Gibson

\subsection{PRESENTACIÓN DEL PROCESO JERÁRQUICO ANALÍTICO}

El Proceso Jerárquico Analítico, conocido como AHP por sus siglas en inglés, fue desarrollado en la década de los 70 por el matemático Thomas L. Saaty, mediante la construcción de un modelo jerárquico, permite de una manera eficiente y gráfica organizar la información respecto de un problema de decisión.
El proceso se fundamenta en varias etapas. La formulación del problema de decisión en una estructura jerárquica es la primera y principal etapa. En esta etapa es en la que el decisor involucrado debe lograr desglosar el problema en sus componentes relevantes. La jerarquía básica está conformada por: meta u objetivo general, criterios y alternativas.

Una vez construida la estructura jerárquica del problema, se da paso a la segunda etapa del proceso de PJA, la valoración de los elementos. El decisor debe emitir sus juicios de valor o preferencias en cada uno de los niveles jerárquicos establecidos. Esta tarea consiste en una comparación de valores subjetivos "por parejas" (comparaciones binarias

El AHP permite realizar las comparaciones binarias basándose tanto en factores cuantitativos (aspectos tangibles) como cualitativos (aspectos no tangibles), ya que presenta su propia escala de medida: la escala $1-9$ propuesta por Saaty y recogida en el cuadro 1.

El resultado de estas comparaciones es una matriz cuadrada, recíproca y positiva

Una vez formadas las matrices de comparación, el proceso deriva hacia la tercera etapa, la fase de priorización y síntesis. El objetivo de esta etapa es calcular la prioridad de cada elemento, entendida esta prioridad tal y como lo define Saaty: La última etapa de este proceso es el denominado análisis de sensibilidad.

La utilización de un software de apoyo, el EXPERT CHOISE, permite resolver el problema de decisión, analizar de forma rápida y sencilla la sensibilidad de los resultados (decisión), a los diferentes cambios posibles, permitiendo analizar el problema en escenarios distintos.

\subsection{PROCESO JERÁRQUICO ANALÍTICO APLICADO A LOCALIZACIÓN DE UNA PLANTA INDUSTRIAL}

Para estructurar el modelo se deben seguir los siguientes pasos:

- Identificación del problema (ubicación geográfica de una planta)

- Definición del objetivo (seleccionar la mejor localidad)

- Identificación de Criterios (factores de localización)

- Identificación de alternativas (localidades a evaluar) 
Cuadro 1. Escala de Medidas de Saaty

\begin{tabular}{|c|c|}
\hline JUICIO SOBRE LA PREFERENCIA & TASA NUMÉRICA \\
\hline Extremadamente más preferido & 9 \\
\hline De muy fuertemente a extremadamente preferido & 8 \\
\hline Muy fuertemente preferido & 7 \\
\hline De fuertemente a muy fuertemente más preferido & 6 \\
\hline Fuertemente más preferido & 5 \\
\hline De moderadamente a poderosamente más preferido & 4 \\
\hline Moderadamente más preferido & 3 \\
\hline De igual a moderadamente más preferido & 2 \\
\hline Igualmente más preferido & 1 \\
\hline
\end{tabular}

Fuente: Tabla propuesta por Saaty y Vargas (1991).

Figura 2. Estructura de PJA.

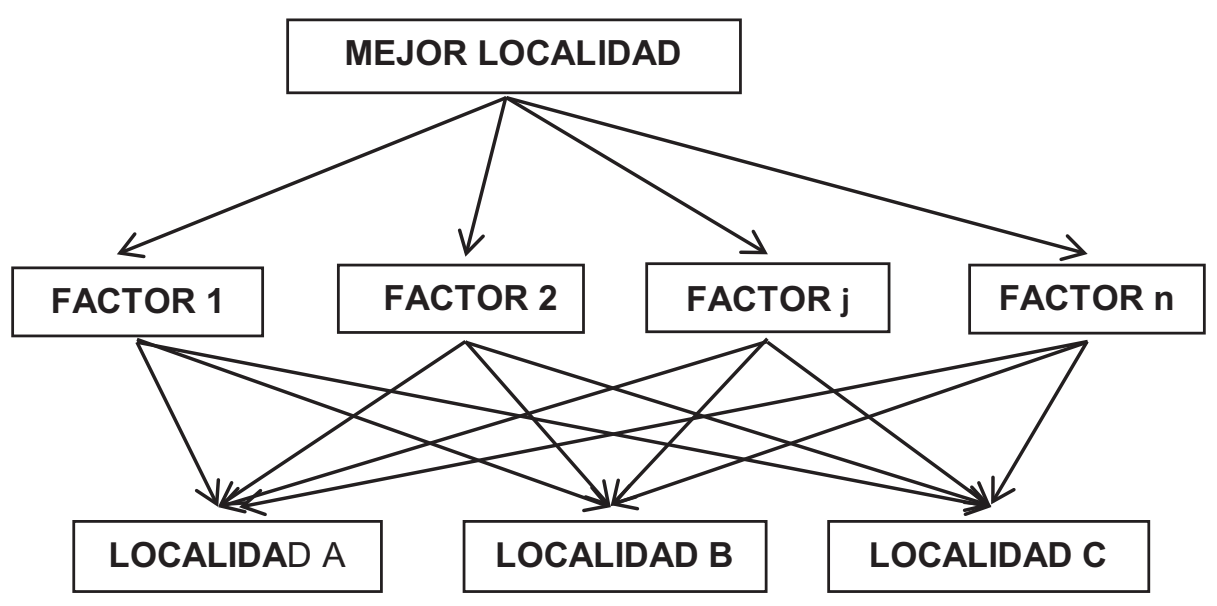

Fuente: Adaptación propia.

\section{PRUEBA DE HIPÓTESIS}

\subsection{ESTRATEGIA DE PRUEBA DE HIPÓTESIS}

Para la prueba de la hipótesis, se aplicaron el modelo Proceso Jerárquico Analítico o AHP (por sus siglas en inglés), así como los modelos del Ranking de Factores, Calificación del Factor Cualitativo, Análisis Dimensional y Brown y Gibson, para seleccionar una planta y luego se compararon los resultados

La comparación de los métodos de selección utilizados, se efectuó mediante la prueba de comparación de medias para más de dos grupos.

\subsection{VERIFICACIÓN DE HIPÓTESIS}

En esta investigación nos planteamos como hipótesis la existencia de una diferencia significativa entre la aplicación de los diferentes métodos para localizar una planta industrial, en los tres casos de estudio.

La consiguiente verificación de las mismas nos condujo a la prueba estadística de comparación de medias mediante el análisis de varianza de un factor y el uso de la distribución $\mathrm{F}$ de Fisher como herramienta para su contrastación.

En el ámbito de la estadística inferencial el criterio para verificar las hipótesis estadísticas de trabajo 
comprende la adopción de un nivel de confianza, en nuestro caso $95 \%$, el cual implica una significancia teórica de $5 \%$, el mismo que debe ser comparado con la significancia obtenida a partir de los datos empíricos para rechazar o aceptar la hipótesis nula. El uso del programa informático SPSS presenta la significancia empírica como valor $\mathrm{p}$ o como Sig en las tablas de resultados. De esta manera cuando el valor $p$ es menor que la significancia teórica $(5 \%=0.05$, en nuestro caso) se rechaza la hipótesis nula y se acepta, por consiguiente, nuestra hipótesis de trabajo.
EI Proceso Jerárquico Analítico en la calificación para la evaluación de los factores cualitativos, optimiza el proceso de localización de una planta inustrial.

Para el caso 1: Tablas de Surf (como ejemplo se muestra los resultados de este)

Ho: Los métodos presentan iguales niveles de decisión respecto a la localización de planta.

H1: Los métodos presentan diferentes niveles de decisión respecto a la localización de planta.

ANOVA de un factor

\begin{tabular}{|c|c|c|c|c|c|}
\hline & $\begin{array}{c}\text { Suma de } \\
\text { cuadrados }\end{array}$ & gl & $\begin{array}{c}\text { Media } \\
\text { cuadrática }\end{array}$ & F & Sig. \\
\hline Inter-grupos & 5616,300 & 3 & 1872,100 & 8,362 &, 008 \\
\hline Intra-grupos & 1791,032 & 8 & 223,879 & & \\
\hline Total & 7407,331 & 11 & & & \\
\hline
\end{tabular}

\subsection{RESUMEN DE LOS RESULTADOS DE LOS CASOS DE APLICACIÓN}

\section{CASO 1: LOCALIZACIÒN DE UNA PLANTA DE SURF}

\begin{tabular}{|c|c|c|c|c|c|c|c|c|}
\hline MODELO & \multicolumn{5}{|c|}{ PRIORIZACIÒN DE LOCALIDADES / PUNTAJE } & CONSISTENCIA & SENSIBILIDAD \\
\hline Ranking de Factores & Trujillo & 85.00 & Piura & 85.00 & Lima & 81.00 & No presenta & No presenta \\
\hline Análisis dimensional & Lima & & Trujillo & & Piura & & No presenta & No presenta \\
\hline $\begin{array}{c}\text { Calificación Factor } \\
\text { Cualitativo }\end{array}$ & Lima & 68.13 & Trujillo & 67.73 & Piura & 61.37 & No presenta & No presenta \\
\hline Brown y Gibson & Lima & 0.61456 & Trujillo & 0.22386 & Piura & 0.16058 & No presenta & No presenta \\
\hline Proceso Jerárquico Anal & Lima & 0.503 & Trujillo & 0.324 & Piura & 0.173 & Si presenta & Si presenta \\
\hline
\end{tabular}

CASO 2: LOCALIZACIÒN DE UN ALMACEN CENTRAL DE UNA EMPRESA MINERA

\begin{tabular}{|c|c|c|c|c|c|c|c|c|}
\hline MODELO & \multicolumn{3}{|c|}{ PRIORIZACIÒN DE LOCALIDADES / PUNTAJE } & CONSISTENCIA & SENSIBILAD \\
\hline Ranking de Factores & Callao & 79.00 & Morococha & 72.00 & La Oroya & 63.00 & No presenta & No presenta \\
\hline Análisis dimensional & Callao & & Morococha & & La Oroya & No presenta & No presenta \\
\hline $\begin{array}{c}\text { Calificación Factor } \\
\text { Cualitativo }\end{array}$ & Callao & 71.22 & Morococha & 53.91 & La Oroya & 51.90 & No presenta & No presenta \\
\hline $\begin{array}{c}\text { Brown y Gibson } \\
\text { Proceso Jerárquico } \\
\text { Analítico }\end{array}$ & Callao & 0.610 & Morococha & 0.237 & La Oroya & 0.153 & Si presenta & Si presenta \\
\hline
\end{tabular}


CASO 3: LOCALIZACIÒN DE UNA PLANTA DE MUEBLES DE OFICINA

\begin{tabular}{|c|c|c|c|c|c|c|c|c|c|c|}
\hline MODELO & \multicolumn{7}{|c|}{ PRIORIZACIÒN DE LOCALIDADES / PUNTAJE } & CONSIS. & SENSIB. \\
\hline $\begin{array}{c}\text { Ranking de } \\
\text { Factores }\end{array}$ & $\begin{array}{c}\text { S. J. } \\
\text { Lurigancho }\end{array}$ & 86.00 & V. El Salvador & 75.00 & Lima Cercado & 71.00 & Ate Vitarte & 65.00 & No pres. & No presen. \\
\hline $\begin{array}{c}\text { Análisis } \\
\text { dimensional }\end{array}$ & $\begin{array}{c}\text { S. J. } \\
\text { Lurigancho }\end{array}$ & & V. El Salvador & & Lima Cercado & & Ate Vitarte & & No pres. & No presen. \\
\hline $\begin{array}{c}\text { Calificación } \\
\text { Fact Cualit }\end{array}$ & $\begin{array}{c}\text { S. J. } \\
\text { Lurigancho }\end{array}$ & 62.50 & Lima Cercado & 52.05 & V. El Salvador & 48.00 & Ate Vitarte & 45.05 & No pres. & No presen. \\
\hline $\begin{array}{c}\text { Brown y } \\
\text { Gibson }\end{array}$ & $\begin{array}{c}\text { Lima } \\
\text { Cercado }\end{array}$ & 0.2901 & $\begin{array}{c}\text { S. J. } \\
\text { Lurigancho }\end{array}$ & 0.2960 & V. El Salvador & 0.2409 & Ate Vitarte & 0.1788 & No pres. & No presen. \\
\hline $\begin{array}{c}\text { Proc } \\
\text { Anal. }\end{array}$ & $\begin{array}{c}\text { S. J. } \\
\text { Lurigancho }\end{array}$ & 0.395 & Lima Cercado & 0.309 & V. El Salvador & 0.173 & Ate Vitarte & 0.122 & Si pres. & Si presen. \\
\hline
\end{tabular}

Descriptivos

\begin{tabular}{|c|c|c|c|}
\hline & $\mathbf{N}$ & Media & Desviación típica \\
\hline 1,00 & 3 & 83,6667 & 2,30940 \\
\hline 2,00 & 3 & 65,7433 & 3,79269 \\
\hline 3,00 & 3 & 33,3367 & 24,55391 \\
\hline 4,00 & 3 & 33,3333 & 16,51979 \\
\hline Total & 12 & 54,0200 & 25,94983 \\
\hline
\end{tabular}

El valor de contraste, $p$, es igual a 0.008 , con el cual, a un nivel de confianza del $95 \%$, se rechaza Ho.

Afirmamos por lo tanto que las diferencias observadas son significativas

Lo mismo sucede con los otros casos de estudio

\section{OBJETIVOS}

Establecer un modelo de calificación para la evaluación de los factores cualitativos, garantizando la optimización del proceso de localización de una planta industrial.

Al probar la hipótesis principal se logró optimizar la calificación de los factores cualitativos, optimizando la localización de planta.

\section{METODOLOGÍA Y TÉCNICAS DE INVESTIGA- CIÓN UTILIZADAS}

Es un tipo de Investigación Aplicada, que se caracteriza por su interés en la aplicación de los conocimientos teóricos propios de la ingeniería industrial, a determinada situación concreta y la evaluación de las consecuencias prácticas que de ellas se deriven, sin embargo, considerando que hecha la revisión de los antecedentes, no se ha podido establecer el uso de dicho modelo en localización, por lo que la investigación también se enmarca dentro del tipo Puro.

\subsection{DISEÑO DE LA INVESTIGACIÓN}

El diseño es No Experimental, Transversal. Es no experimental en la medida en que no se manipula la variable independiente, porque los efectos se aprecian en el largo plazo (20 años o más). Es transversal por que el análisis para la localización de una planta industrial se realiza en un periodo específico de tiempo, no a través del tiempo

La naturaleza de la investigación predispone la utilización del diseño Descriptivo Correlacional, el mismo que permitirá determinar como la aplicación del modelo Proceso Jerárquico Analítico optimiza el proceso de selección de una planta industrial. También se considera una investigación Exploratoria por la búsqueda de información para la aplicación al proceso de localización. 


\subsection{POBLACIÓN Y MUESTRA}

Por la naturaleza del objeto de estudio, la población se circunscribe al caso o casos seleccionados, sobre los cuales se aplican los modelos anteriormente mencionados, comparándolo con el modelo Proceso Jerárquico Analítico.

La muestra es igual a la población, es decir se estudiaron los casos seleccionados.

\section{CONCLUSIONES}

1. El Proceso Jerárquico Analítico, en comparación con los otros modelos de localización de planta, es un modelo multicriterio que permite una adecuada evaluación de los factores cualitativos, optimizando el proceso de localización de una planta industrial.

2. El Proceso Jerárquico Analítico, tiene una escala de calificación que permite la disminución del grado de subjetividad, en la evaluación de los factores cualitativos.

3. El Proceso Jerárquico Analítico, permite una adecuada ponderación de los factores locacionales entre sí, mejorando los criterios de localización de una planta industrial.

4. El Modelo de Proceso Jerárquico Analítico, con su RELACIÓN DE CONSISTENCIA, garantiza la correcta calificación de los factores locacionales, corrigiendo la inconsistencia que se puede presentar en los otros modelos de localización. Así mismo permite realizar un ANÁLISIS DE SENSIBILIDAD, al variar las condiciones de los factores locacionales.

5. El software EXPERT CHOICE, garantiza el manejo adecuado del modelo de Proceso Jerárquico Analítico para una localización de una planta industrial.

6. Los resultados parciales del Proceso Jerárquico Analítico, permiten: Priorización de los factores locacionales y priorización de localidades por cada factor

\section{REFERENCIAS BIBLIOGRÁFICAS}

[1] Ávila Mogollón, Ruth Maritza (2000). "El AHP (Proceso Analítico Jerárquico) y su Aplicación para determinar los Usos de las Tierras". El caso de Brasil. Proyecto GCP/RLA/126/JPN.

[2] Chase; Aquilano, Jacob (2000). Administración de Producción y Operaciones. Mc Graw Hill. Bogotá-Colombia. 885 pgs.
[3] Cock, German; Lopez Rendón, Juan Fernando (2009). Ingenieros Industriales Profesores de la Universidad Tecnológica de Pereira: "Lúdica aplicada al problema de localización de planta física utilizando Analytic Hierachy Proces (AHP) y Solver para Excel".

[4] Díaz Garay Bertha; Jarufe Zedán Benjamin; Noriega Aranibar María (2001). Disposición de Planta. Universidad de Lima-Fondo Editorial. Lima- Perú. 344 pgs.

[5] Fernández PIrla, J.(1981): Economía y gestión de la empresa, ICE, Madrid,

[6] García Cascales, María del Socorro (2009): Métodos para la Comparación de Alternativas mediante un Sistema de Ayuda para la Decisión. S.A.D. Soft Computing". Tésis para optar el grado de Doctor en la Universidad Politécnica de Cartagena España.

[7] Heizer Jery; Render Barry (1998). Dirección de la Producción. Prentice Hall. BarcelonaEspaña. 576 pgs.

[8] Hernández, Fernando (2009). Factores determinantes que marcan una ventaja competitiva del lugar en la localizacion de futuros emplazamientos empresariales en la frontera noreste de México". http://www. eumed.net/cursecon/ecolat/mx/2009/cimgf. htm. Consultado Diciembre 2011

[9] Konz Stephan (1992). Manual de Distribución en Plantas Industriales. Grupo Noriega Editores. México. 127 pgs.

[10] Krajewski Lee J.; Ritzman Larry P. (2000). Administración de Operaciones, Estrategia y Análisis. Prentice Hall. México. 740 pgs.

[11] Márquez Rosales, Heliodoro (2009). "El Riesgo Arquelógico en Andalucía. Una Evaluación a Través del Modelo de Jerarquía Analítica de T. L. Saaty y los Sistemas de Información Geográfica".

[12] Miranda Miranda, Juan José (2008). Localización Final de Una Planta.

[13] Nahmías S. (1999). Análisis de la Producción y la Operaciones. CECSA. México. 817 pgs.

[14] Pérez León, Víctor; Camargo Toribio, Isis; Caballero Fernandez, Rafael y Gonzales Lozano, Mercedes (2008): "Selección Multicriterio de Nuevos Productos Turísticos en Pinar del Río Cuba". Revista de Investigación Operacional Vol 2, Nº 2, 98-107, 2008 
[15] Phillips Edward J. (1997). Manufacturing Plant Layout. Society of Manufacturing Engineers (SME). E.E.U.U. 259 pgs.

[16] Ramirez, Mirta Liliana (2004): "El Método de Jerarquías Analíticas de Saaty en la Ponderación de Variables. Aplicación al Nivel de Mortalidad y Morbilidad en la Provincia del Chaco Argentina". Universidad Nacional del Nordeste.

[17] Render, Barry: Heizer, Yay (2007): Principios de la Administración de Operaciones.

[18] Reséndiz Vásquez, Héctor (2009): “Diseño de una Planta Productora de Lombricomposta en la Universidad Tecnológica de la Mixteca", Tésis para optar el Título de Ingeniería Industrial. México

[19] Saaty, Thomas (1997). Toma de Decisiones para Líderes: El Proceso Analítico Jerárquico la Toma de Decisiones en un Mundo Complejo/
Traducción Mauricio Escudey, Eduardo Martinez Y Luis Vargas

[20] Sack Nigel; Chambers Stuart (1999). Administración de Operaciones. Cía Editorial Continental. México. 864 pgs.

[21] Toskano Hurtado, Gérard Bruno (2005): "EI Proceso de Análisis Jerárquico (AHP) como Herramienta par la Toma de Decisiones en la Selección de Proveedores". Tésis para optar el Título de Licenciado en Investigación de Operaciones de la UNMSM. SISTEMA DE BIBLIOTECAS.

[22] Vásquez Barquero, A (1988). "Las iniciativas locales y los sistemas de localización industrial". Revista Estudios Regionales, №22, pp 69-82.

[23] Von Thünen, Johann Heinrich (1826). El modelo "La Teoría de la Localización". "Model of Agricultural Land Use". "Las Decisiones de Localización en las Actividades Agrícolas" Wikipedia. 